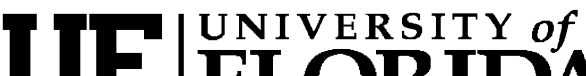 FLORIDA \\ IFAS Extension
}

\section{Swarm Trapping for Pest Control Operators ${ }^{1}$}

\author{
M. K. O'Malley, J. D. Ellis and C. M. Zettel Nalen ${ }^{2}$
}

\section{Introduction}

African honey bees were first documented in Florida in 2002. Since then, the risk of Africanized bees infiltrating urban environments has increased as the Africanized bee population has increased. Swarm trapping by a certified Pest Control Operator provides Floridas citizens a means of capturing and removing a nesting bee colony before the bees become aggressive.

The Florida Department of Agriculture and Consumer Services recommends that only trained Pest Control Operators engage in swarm trapping. Despite the increasing need for swarm trapping, very few pest control companies currently offer this service. This document outlines the standard operating procedures for swarm trapping. Please note, these procedures are NOT meant for homeowners and should be conducted by trained and licensed Pest Control Operators only.

\section{Swarms and Trapping}

Honey bee colonies reproduce by increasing the number of individual bees, then dividing the colony. As a colony divides, approximately $60 \%$ of the hive will leave the nest with the existing queen in search of a new nest location. This group of bees leaving the nest is known as a swarm.

Most people use the term "swarming" to refer to dangerous bee activity or foraging bees flying around; however, this is not accurate. A swarm is a condensed body of bees concentrated in a specific area or moving from its previous colony to a temporary location until they find a permanent nest site. Ultimately, swarming allows honey bees to increase their abundance in the environment. European honey bees will swarm once or twice a year, however Africanized honey bees can swarm up to 10 times a year.

Upon leaving the original colony, the queen will settle on a nearby structure (often a tree branch, side of a house, etc.). At this time, the workers that followed her circle in the air above her and land on and around her to protect her. The swarm can range from the size of an orange to the size of a five gallon bucket.

The swarm cluster is only a temporary location for the bees. Scout bees will leave the swarm and search for new suitable nest locations. Once the scout

1. This document is ENY-148 (IN785), one of a series of the Entomology \& Nematology Department, Florida Cooperative Extension Service, Institute of Food and Agricultural Sciences, University of Florida. First published: October 2009. For more publications related to horticulture/agriculture, please visit the EDIS Website at http://edis.ifas.ufl.edu/.

2. M. K. O'Malley, former extension assistant, J. D. Ellis, assistant professor, C. M. Zettel Nalen, extension assistant, Entomology \& Nematology Department, Institute of Food and Agricultural Sciences, University of Florida, Gainesville, FL 32611.

The Institute of Food and Agricultural Sciences (IFAS) is an Equal Opportunity Institution authorized to provide research, educational information and other services only to individuals and institutions that function with non-discrimination with respect to race, creed, color, religion, age, disability, sex, sexual orientation, marital status, national origin, political opinions or affiliations. U.S. Department of Agriculture, Cooperative Extension Service, University of Florida, IFAS, Florida A. \& M. University Cooperative Extension Program, and Boards of County Commissioners Cooperating. Millie Ferrer-Chancy, Interim Dean 
bees locate a potential nest site, they leave the group and move into their new nest, where soon the other bees will follow.

Bees in swarms are generally docile and not defensive as they have not yet established resources to defend. Despite their passive demeanor, swarms should be removed while they are vulnerable and not yet in a difficult location where they can potentially become aggressive or cause structural damage.

Swarm trapping is a precautionary measure that captures swarms before they find a nesting site from which they may be difficult to remove. Swarm trapping is done by setting out traps in strategic locations, and attracting the bees with pheromone lures as they search out a suitable nest location.

\section{Equipment}

Swarm trapping requires few specialized tools. Swarm traps are 5-10 gallon pots, consisting of a body and a lid, made from a fiber/pulp-paper based material. Often, they are treated with a fungicide to prevent mold and rot if exposed to weather. Traps cost about $\$ 15.00$, and lures about $\$ 3.00$. Lures come in small plastic vials and are filled with a liquid pheromone which attracts the bees. Lures can be purchased from most beekeeping supply companies. Additional recommended equipment: safety cones, safety vest, rubber gloves, first aid kit, soap \& water, hard hat, splash goggles, binoculars, beekeeping protective gear, hive smoker, weather-proof labeling tags, a spool of wire, wire cutters, garbage bags, duct tape, hammer, nails, staple gun, staples, and pesticide foams (soapy water also works well for swarms-mix 1 cup of liquid detergent to 3 gallons of water).

\section{Placement}

Generally, swarm traps (also called bait hives) should be placed along the perimeter of a client's property, about 50-100 yards apart. When they are placed in such a fashion, the string of traps is referred to as a trap line. When considering swarm trap locations, remember that the traps should be placed away from homes or other buildings frequented by people. The traps should be placed $10-20$ feet high if possible in a shady area, yet they need to be easily accessible for the removal of bees. Placement of traps should allow room to park and exit vehicles at a safe distance, yet traps should not be easily accessible to the general public. Be aware of environmental hazards while placing traps such as power lines, unstable trees, etc. During placement, each trap should be labeled with the trap-line name, date last worked, and contact information. Survey records and maps should be maintained for each trap site.

\section{Assembly}

The traps should be assembled on site, so be sure to take precautions while parking or working near a public right-of-way. Always display safety cones and wear a safety vest. Be aware that some construction sites and port areas require hard hats. To assemble traps, first insert wiring through the center of the trap for added support (this also provides an anchor site that can be used for hanging) (Figures 1 and 2).

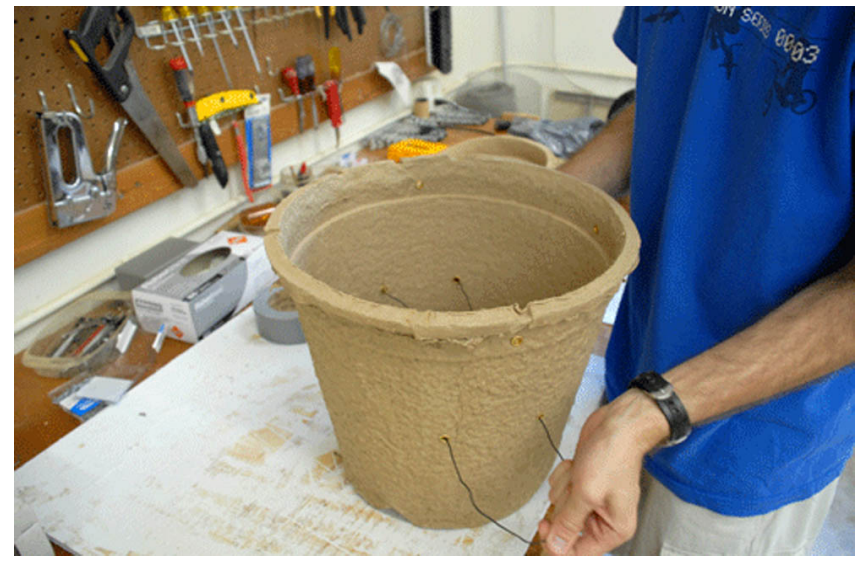

Figure 1. Wiring through center of trap. Credits: M.K. O'Malley, University of Florida

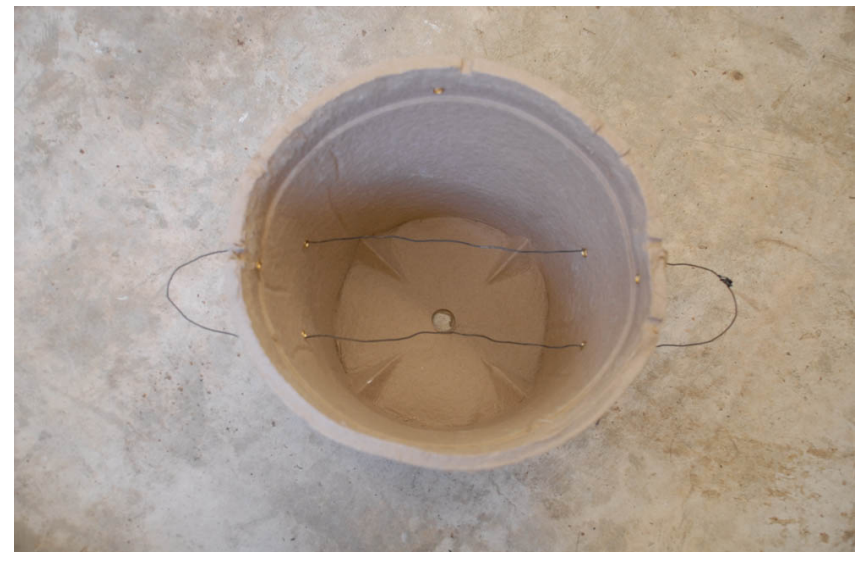

Figure 2. Wiring through center of trap. Credits: M.K. O'Malley, University of Florida 
Next, cut two pieces of wire, approximately three inches in length. Use a staple gun to secure wire in a cross-shape over the traps opening; this will help discourage intruders (Figure 3).

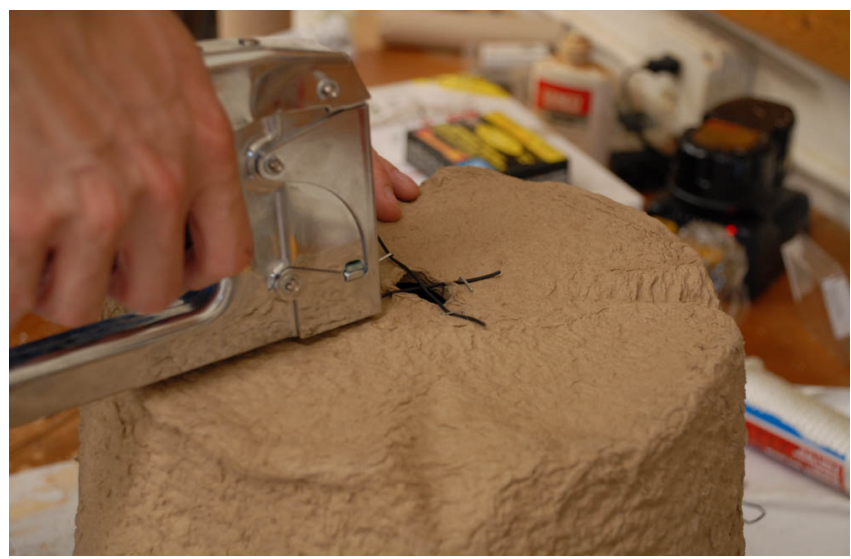

Figure 3. Staple two pieces of wire in the shape of a cross across small trap opening. Credits: M.K. O'Malley, University of Florida

Next, a wire hook or hanger should be created to hang the pheromone lure (Figure 4).

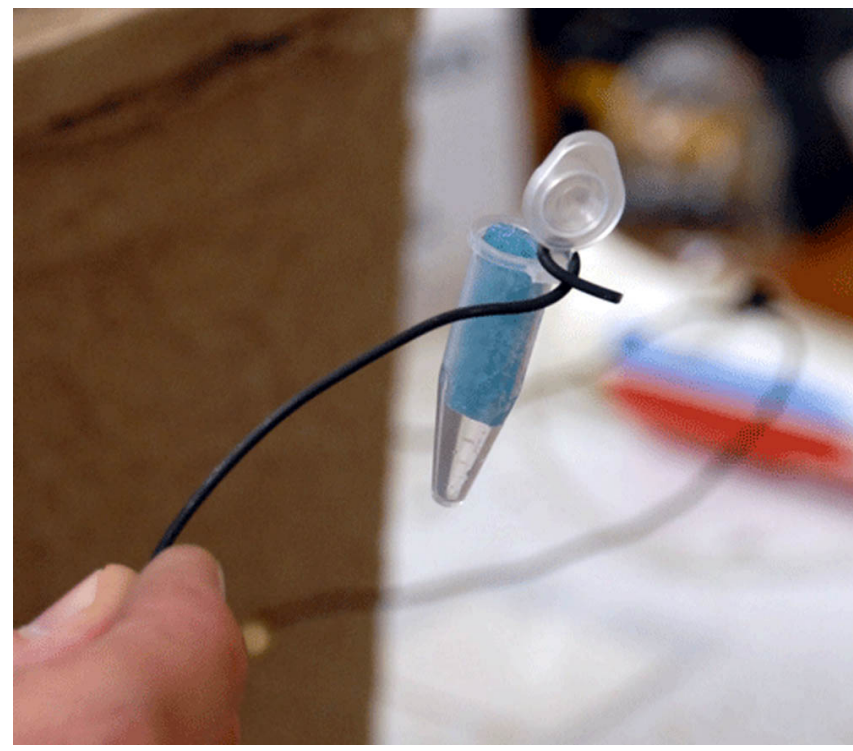

Figure 4. Construct a pheromone lure hanger. Credits: M.K. O'Malley, University of Florida

Once the hanger is made, hang the lure on the inside of the trap's opening, and staple the protruding wire to outside (Figures 5).

Finally, nail together the trap top and bottom (Figure 6).

Because it must be able to support the weight of a swarm, be sure that the trap is firmly attached to an

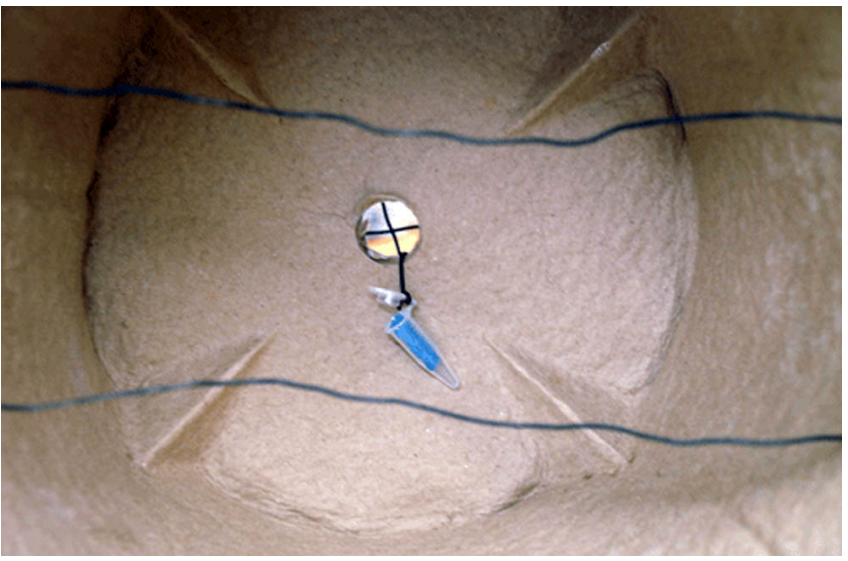

Figure 5. Hang the pheromone lure on inside of the trap opening. Credits: M.K. O'Malley, University of Florida

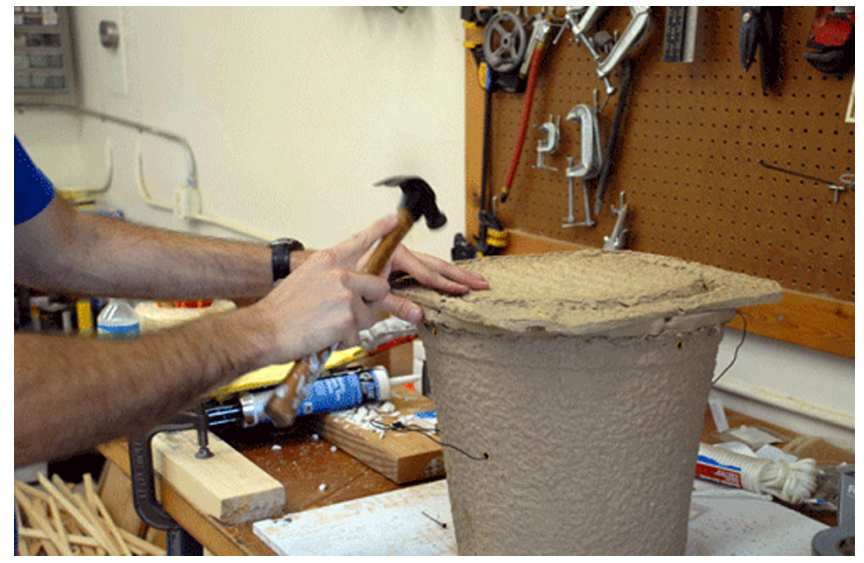

Figure 6. Nail the top and bottom of the trap together to provide a dark space for the bees. Credits: M.K. O'Malley, University of Florida

object (usually a tree, but other structures can be used if necessary). The trap can be hung using wire or rope, or attached to a surface with screws-just be sure it will handle the weight of a colony of bees, comb, and honey ( 10-30 lbs.). If hanging traps in trees, placing the traps $10-20$ feet above ground is ideal. Once the trap is hung, fill out and attach an information card (Figures 7,8).

\section{Servicing}

Traps should be monitored every 21 days, and pheromone lures should be replaced twice a year in January/February and July/August. Traps should be placed high enough that binoculars can be used to determine if any swarms have been trapped. Placing traps at such a height allows Pest Control Operators or homeowners to monitor for bees without putting themselves in danger of a defensive attack. If bee activity is noticed in or around a trap, the colony must 


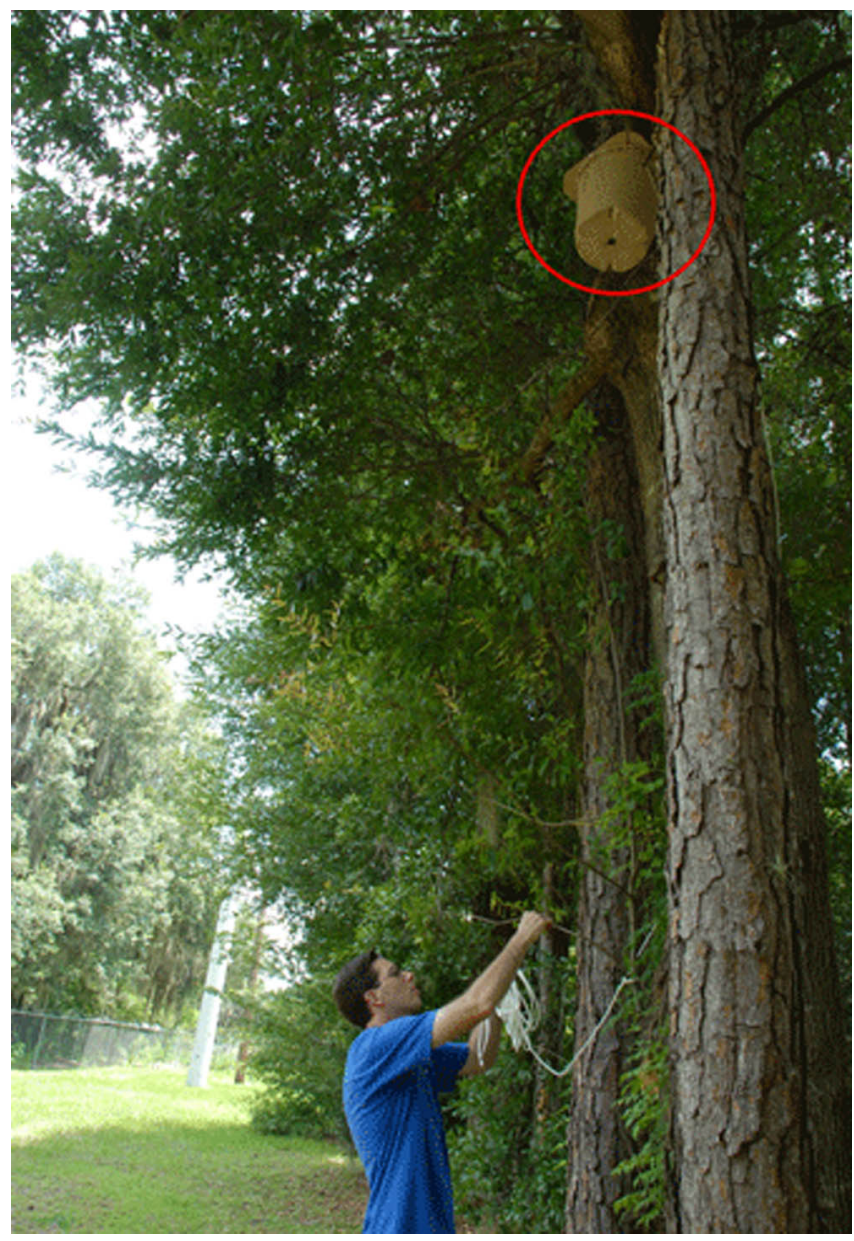

Figure 7. Trap secured with rope between 10 to 20 feet above ground. Credits: M.K. O'Malley, University of Florida

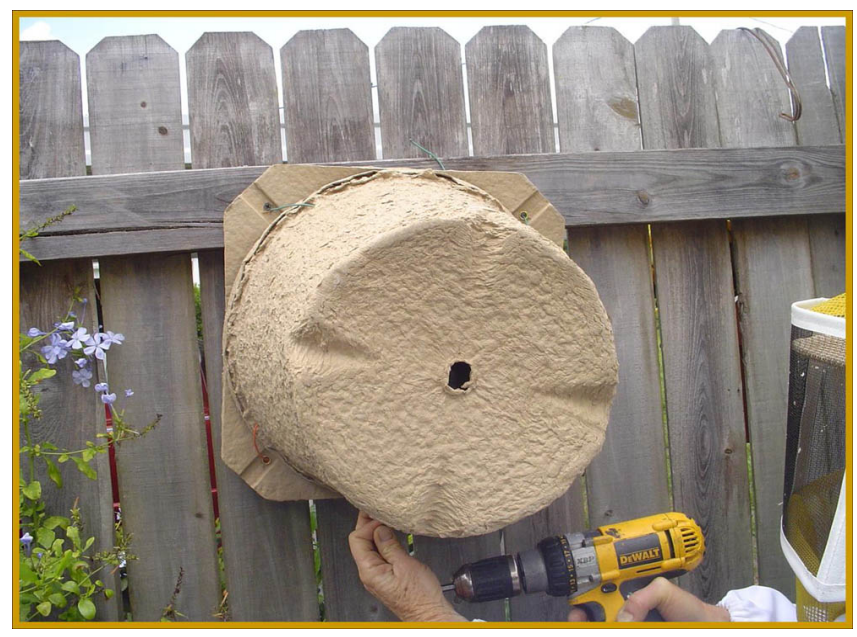

Figure 8. Traps can also be placed on other structures depending where the swarm is located. Credits: M.K. O'Malley, University of Florida

be removed immediately. Any bees caught by the trap may exhibit defensive characteristics; therefore, a complete beekeeper protective suit should be worn (to prevent openings in suit, pants can be duct taped to boots, and gloves taped to suit).

After securing the bee suit, light the smoker, approach the trap with caution, slowly lower the trap, and inject smoke into the traps opening (Figure 9).

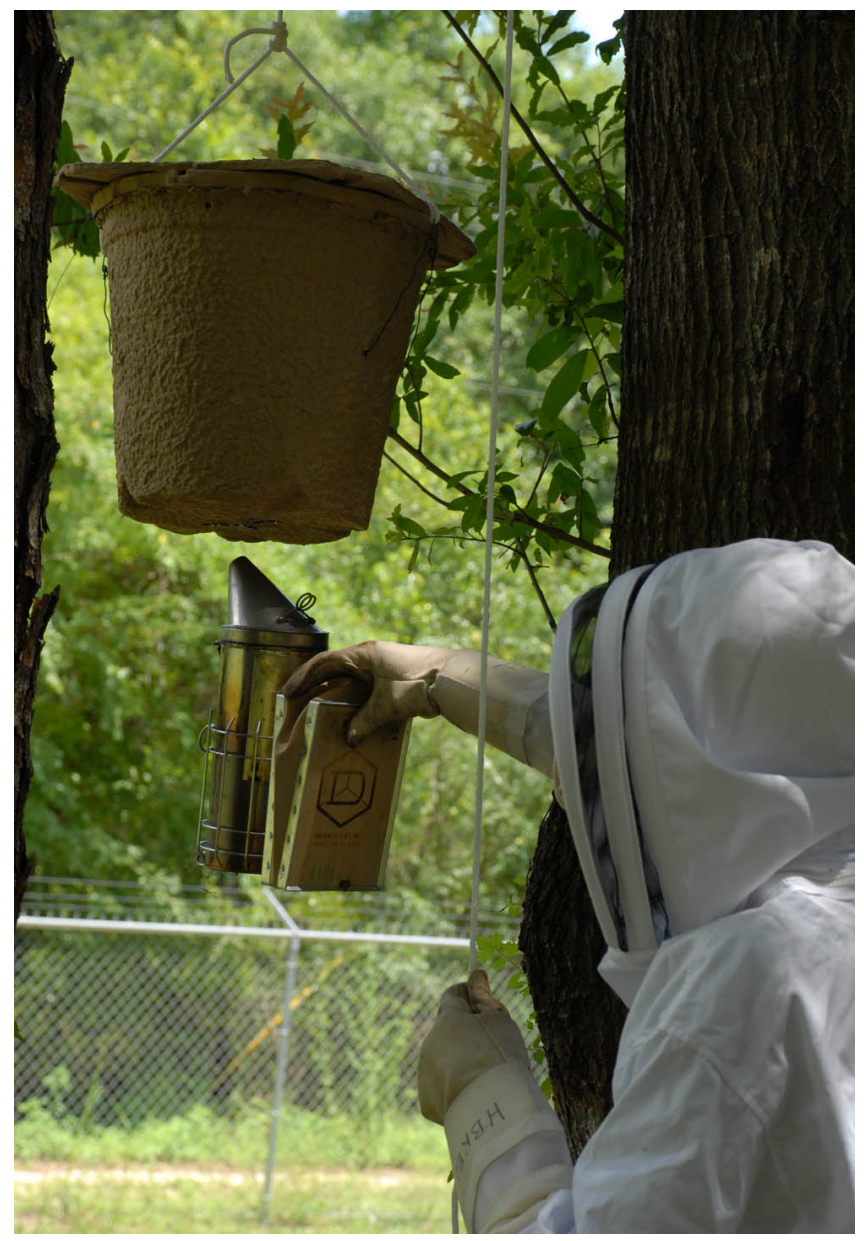

Figure 9. Injecting smoke into the opening of the trap to mask the honey bee alarm pheromone. Credits: M.K. O'Malley, University of Florida

Gently remove the trap from the structure, place the entire trap in a garbage bag and seal the bag with duct tape (Figure 10).

Once the trap is in the bag, there are several options for eliminating the colony. The trap entrance can be located through the bag, and a pesticide sprayed through the bag into the opening (Figure 11). Other options include placing the hive in a freezer for several hours to freeze the bees, or leaving the bag in full sun for several hours to solarize the bees. Label the bag with the date, location and other details as needed. If the client is interested in getting the bees 


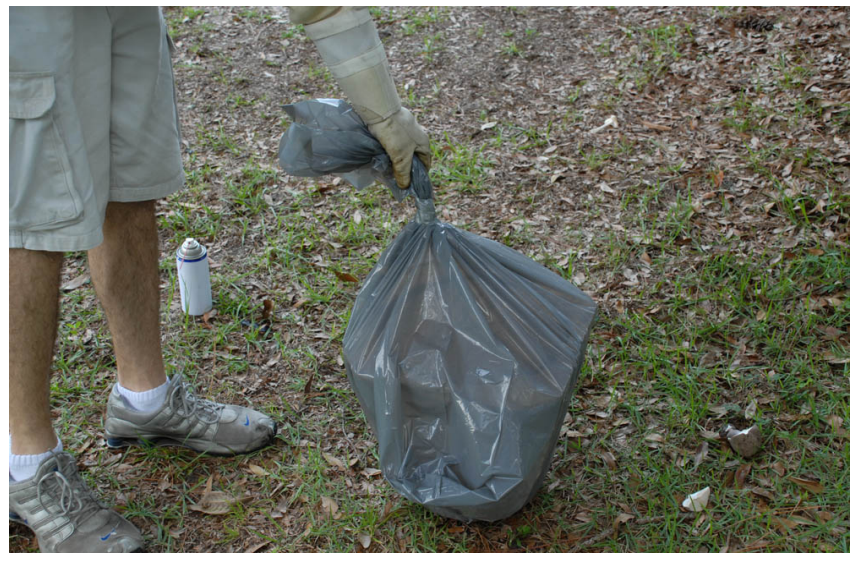

Figure 10. Trap in bag sealed with duct tape Credits: M.K. O'Malley, University of Florida

tested to determine if they are Africanized, samples of approximately 50 bees preserved in alcohol can be sent to the Florida Department of Agriculture and Consumer Services.

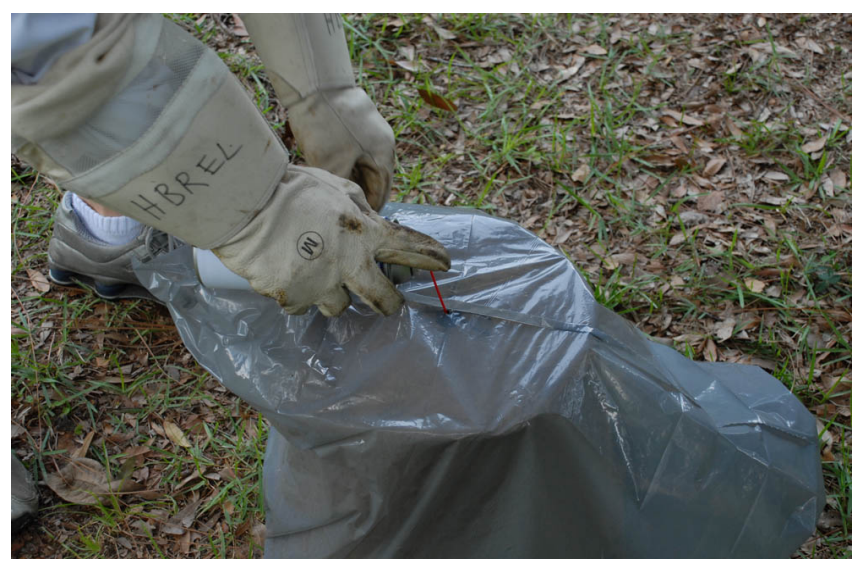

Figure 11. Spraying pesticide through the bag into the trap's opening Credits: M.K. O'Malley, University of Florida

Remember to always thoroughly wash your hands after using pesticides.

\section{Proposed Business Plans}

Pest control operators may choose to include swarm trapping as a part of their Africanized honey bee removal and monitoring services. Three grades of service are suggested:

\section{Trapping Station Installation-initial visit}

- Property analyzed by pest control personnel

- Number and location of necessary traps determined
- Each trap is installed and outfitted with pheromone lure

- Per-trap charge recommended

Premium Service Plans_-site maintenance

- Pest control personnel inspect stations every 21 days

- Trapped swarms neutralized and removed

- Monthly charge recommended

Self-Monitoring Service Plan—site maintenance

- Clients inspect stations regularly

- Client contacts company when a swarm is trapped

- Personnel neutralize and remove each swarm

- Monthly per-trap charge recommended plus additional charge for each removed swarm

\section{Standard Operating Procedure Summary}

\section{Trap assembly:}

1. Insert wiring through the center of the trap for added support while hanging

2. Use staple gun to attach wire in a cross-shape over the opening in the trap; this will help discourage intruders

3. Create a hanger for the pheromone lure using wire

4. Hang lure on the inside of the trap's opening, and staple protruding wire to outside of trap

5. Nail together the trap top and bottom

6. Trap lines should be established along perimeters of property

7. Trap should be placed as high as possible (10-20 ft.)

8. Because it must be able to support the weight of a swarm, be sure to attach the trap securely to a tree or other object 
9. Attach the information tag to the trap

\section{Trap servicing:}

Monitor traps on 21-day intervals, change pheromone lures in July/August and January/February

1. Park vehicle a safe distance from trap in order to prepare for bee removal

2. Due to the unpredictable and defensive nature of African honey bees, put on complete protective gear (veil, jacket/pants, gloves, boots)

3. Light smoker

4. Approach bait hive with caution, and inject smoke into the opening

5. Detach and lower trap

6. Place trap in garbage bag, and seal bag with duct tape

7. Locate trap opening and spray in pesticide (foams work best)

8. Label bag for sampling

9. Wash hands with soap and water after using pesticides

\section{Equipment list:}

1. Trap top and bottom

2. pheromone lure

3. safety cones

4. safety vest

5. rubber gloves

6. first aid kit

7. soap \& water

8. hard hat

9. splash goggles

10. beekeepers protective gear
11. hive smoker

12. binoculars

13. weather-proof labeling tags

14. spool of wire

15. wire cutters

16. garbage bags

17. duct tape

18. hammer

19. nails

20. staple gun

21. staples

22. pesticides (soapy water works well for swarms - mix 1 cup liquid detergent to three gallons of water) 